\title{
Comparison of storage phosphor computed radiography with conventional film-screen radiography in the recognition of pneumoconiosis
}

\author{
A.S. Laney, E.L. Petsonk, A.L. Wolfe and M.D. Attfield
}

ABSTRACT: Traditional film-screen radiography (FSR) has been useful in the recognition and evaluation of interstitial lung diseases, but is becoming increasingly obsolete. To evaluate the applicability of storage phosphor digital computed radiography (CR) images in the recognition of small lung opacities, we compared image quality and the profusion of small opacities between FSR and CR radiographs.

We screened 1,388 working coal miners during the course of the study with FSR and CR images obtained on the same day from all participants. Each traditional chest film was independently interpreted by two of eight experienced readers using the International Labour Office (ILO) classification of radiographs of pneumoconiosis, as were CR images displayed on medical-grade computer monitors.

The prevalence of small opacities (ILO category $1 / 0$ or greater) did not differ between the two imaging modalities (5.2\% for FSR and $4.8 \%$ for soft copy CR; $p>0.50)$. Inter-reader agreement was also similar between FSR and CR. Significant differences between image modalities were observed in the shape of small opacities, and in the proportion of miners demonstrating high opacity profusion (category $2 / 1$ and above).

Our results indicate that, with appropriate attention to image acquisition and soft copy display, CR digital radiography can be equivalent to FSR in the identification of small interstitial lung opacities.

\section{KEYWORDS: Imaging, pneumoconiosis}

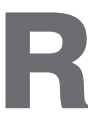

outine chest radiography has been an essential tool in research and in the recognition of interstitial lung diseases and continues to be recommended to monitor lung health among individuals with potential exposures to toxic dusts [1]. However, growing numbers of clinical facilities are abandoning conventional filmscreen radiography (FSR) and adopting digital technologies for routine chest imaging. Computed tomography (CT) for imaging the chest has also become widely available, and is more sensitive for demonstrating interstitial changes. However, $\mathrm{CT}$ involves both higher radiation exposures and greater expense, and thus is not considered appropriate for periodic screening in generally healthy populations. Digital chest imaging appears to be rapidly replacing FSR for the routine monitoring of lung health among individuals at risk for interstitial lung disorders.

Few studies have addressed whether the newer commercially available digital radiography systems are equivalent or superior to traditional FSR in the recognition of subtle interstitial abnormalities in patients and exposed workers. Presently, two basic technologies are used to acquire digital plain chest images: storage phosphor computed radiography $(\mathrm{CR})$ and digital direct readout radiography (DR) systems [2]. One previous study evaluated DR chest images and confirmed their utility with respect to the recognition and classification of pneumoconioses [3]. Because CR systems are in widespread use but have not been extensively assessed, we evaluated radiographic opacities among 1,388 dust-exposed workers using CR digital chest images displayed on medical-grade computer monitors, as well as hard copy FSR taken on the same day.

\section{MATERIALS AND METHODS}

The US National Coal Workers' X-ray Surveillance Program (CWXSP) has been extensively described elsewhere, with respect to its historical

\section{AFFILIATIONS}

Surveillance Branch, Division of Respiratory Disease Studies, National Institute for Occupational Safety and Health, Centers for Disease Control and Prevention, Morgantown, WV, USA.

\section{CORRESPONDENCE}

A.S. Laney

Surveillance Branch, Division of

Respiratory Disease Studies, National Institute for Occupational Safety and Health

Centers for Disease Control and Prevention

1095 Willowdale Road

Mail Stop HG900.2

Morgantown

WV 26505-2888

USA

E-mail: alaney@cdc.gov

Received:

Aug 102009

Accepted after revision:

Nov 022009

First published online:

Nov 192009 
perspectives, methods and findings [4-6]. The data for this study were derived from an extension of that programme: the Enhanced Coal Workers' Health Surveillance Program (ECWHSP). This employs a mobile examination unit that visits coal mining regions for the purpose of investigating previously recognised "hotspots" of coal workers' pneumoconiosis (CWP) [7]. Underground miners were recruited to provide a CR digital image in addition to the standard FSR, based upon their eligibility for participation in the CWXSP. The National Institute for Occupational Safety and Health (NIOSH; Morgantown, WV, USA) institutional review board approved the project, and each study participant gave written informed consent.

\section{Subjects}

FSR and CR images were acquired on the same day from 1,401 miners between February and September 2007. Images from miner participants were consecutive (all images contributed by participants during the study period were included for analysis). Classifications from 13 miners were excluded from the analysis because at least one reader classified a radiograph's quality as unreadable. The remaining 1,388 miners contributed one FSR and one CR image for this study. All participants were males. Mean age was 48.1 yrs on the date of the examination.

\section{Image acquisition}

Standard posterior-anterior FSR and CR images were obtained using the same X-ray source, a Toshiba X-ray tube model number E7252X (Toshiba, Otawara, Japan) with a 72-inch source to image distance and a $14 \times 17$-inch cassette with a mobile Bucky grid. A standard voltage of $110 \mathrm{kVp}$ was used. $\mathrm{X}$-ray tube current settings in $\mathrm{mAs}$ were manually selected from a standard exposure chart using recommended values based upon body habitus and callipers measurement of chest diameter. CR images were captured with an Agfa phosphorbased image plate (AGFA Corporation, Ridgefield Park, NJ, USA), with mAs generally twice that used for FSR.

\section{Image processing}

Images were processed immediately after each examination. FSR images were developed in the mobile unit using an Agfa ${ }_{\mathbb{R}}$ model CP 1000 film processor with Agfa G-153 developer and G-353C fixer (AGFA Corporation). The latent CR image was transferred from the plate to a computer file by processing the cassette through an Agfa CR 25.0 digital image reader. The CR image files were generated using Agfa Musica ${ }^{\circledR}$ automated digital radiographic image processing software (AGFA Corporation), then reviewed at a quality assurance work station and stored on the network hard drive of the mobile examination unit.

\section{Image reading}

Eight NIOSH-approved B readers provided the International Labour Office (ILO) pneumoconiosis classifications for the study [1]. Each reader was assigned a subset of the 1,388 paired images (FSR and CR). Readers always interpreted both of the paired images from an individual miner, but the FSR and CR images were presented at separate reading sessions, and readers were blinded to the results of their own or other readers' previous interpretations. To account for possible differences due to reading practices, readings were analysed by pairs of readers (reader A with reader B, A with C, B with C, etc.), with a total of 28 possible reader pairings. The number of image pairs assigned to each reader pair ranged from 40 to 72 .

FSR images were displayed on a standard two-gang X-ray view box side-by-side with the selected ILO film-based standard. CR images were displayed using dual-screen highresolution physician-quality workstations, with resolution $2048 \times 2560$ pixels, and maximum luminance of $10 \mathrm{~cm} \times 10 \mathrm{~cm}$ area $600 \mathrm{~cd} \cdot \mathrm{m}^{-2}$, and of a fully white screen $350 \mathrm{~cd} \cdot \mathrm{m}^{-2}$. Brightness and contrast of the monitors were calibrated to satisfy the Digital Imaging and Communications in Medicine $\left(\mathrm{DICOM}_{\ltimes}\right)$ grayscale standard display function. The image display devices met the specifications for display capabilities of the large-matrix size diagnostic monitors as specified by the American College of Radiology Technical Standard for Electronic Practice of Medical Imaging 2007 and the DICOM standard 3.14 for grayscale display function [8]. Classifications were performed using side-by-side comparisons with digitised versions of the ILO standard films that had been previously validated [3]. Readers were instructed not to modify the display characteristics of the digitised standard images during the classification process, but were permitted to modify brightness, contrast, and magnification of the miner's digital chest radiograph on the workstation, as permitted in the study of FRANZBLAU et al. [3]. This approach preserved the commonality of the set of ILO standard images while permitting the reader to more closely match the display characteristics of the miner's chest radiograph with those of the standard image(s) selected for comparison. Image modifications, if any, were not recorded.

\section{Data analysis}

Kappa statistics were employed to examine inter and intrareader agreement. For contingency tables larger than $2 \times 2$, Cicchetti-Allison weighted kappas were used [9]. The Wilcoxon rank sum test statistic was used to compare median kappa values between modalities. The $S A S_{\circledR}$ statistical software package version 9.1 (SAS Institute, Cary, NC, USA) was used for all analyses.

\section{RESULTS}

In total, 1,388 miners contributed one FSR and one CR image. Each image was interpreted by two NIOSH-approved B readers yielding 5,552 total readings (2,776 for each modality). Information on reported image quality is presented in table 1 by CR and FSR. Significantly more images were classified as category 3 and 4 (some technical defects or unacceptable quality for classification purposes) for FSR compared to $\mathrm{CR}$ $(\mathrm{p}<0.001)$.

\section{Differences between modalities}

The distribution of the profusion of small opacities for the 1,388 radiographs is shown by modality in figure 1 . Of the 2,776 FSR readings, $91.3 \%$ (2,535 out of 2,776) were classified as profusion category $0 / 0$. For the CR images, $92.1 \%$ were classified as $0 / 0$. Of the 1,388 image pairs, $78.9 \%(1,095$ out of 1,388 ) were classified in full agreement (first reader FSR = first reader $\mathrm{CR}=$ second reader $\mathrm{FSR}=$ second reader $\mathrm{CR}$ ). In all instances of complete agreement the classification was $0 / 0$. 


\begin{tabular}{|c|c|c|}
\hline Quality & FSR & Digital CR \\
\hline 1) Good & $1735(61.9)$ & $2032(72.5)$ \\
\hline 2) Acceptable, no defects & $701(25.0)$ & $621(22.2)$ \\
\hline 3) Acceptable, some defects & $335(12.0)$ & $129(4.6)$ \\
\hline 4) Unacceptable & $13(0.46)$ & 0 \\
\hline 5) Did not rank quality ${ }^{\#}$ & $18(0.64)$ & $20(0.71)$ \\
\hline \multicolumn{3}{|c|}{$\begin{array}{l}\text { Data are presented as } n(\%) \text {. Includes initial 1,401 miner participants. Image } \\
\text { quality assessed by two B readers for film-screen radiography (FSR) and digital } \\
\text { storage phosphor computed radiography (CR) images. The } 13 \text { unacceptable } \\
\text { quality images were from } 13 \text { miners and all images obtained from those miners } \\
\text { were excluded from subsequent analyses. }{ }^{*} \text { : data for other fields, including } \\
\text { small opacity profusion characterisations, were complete. }\end{array}$} \\
\hline
\end{tabular}

The total number of radiographs classified as abnormal (profusion category $1 / 0$ or greater) was similar between FSR $(5.2 \%)$ and CR (4.8\%) with a Chi-squared p-value of 0.54 . However, more images were classified as profusion category $2 / 1$ or greater by CR $(1.0 \%)$ compared with FSR $(0.40 \%$; $\mathrm{p}=0.006)$. The $C R$ category $2 / 1$ or greater images tended to be read as $1 / 2$ or $1 / 1$ using FSR (table 2). In table 2 , the overall weighted kappa was 0.49 (95\% CI 0.44-0.54), denoting good agreement. When small opacity profusion was treated dichotomously $(0 / 0$ or $0 / 1$ versus profusion $1 / 0$ or greater), intermodality agreement increased $(\kappa 0.70,95 \%$ CI $0.62-0.77)$. There were too few participants demonstrating large pneumoconiotic opacities (progressive massive fibrosis) for reliable comparison of modalities, with only 10 radiographs showing large opacities by FSR and nine by CR. Coalescence of small opacities was classified by readers in six FSR images and eight CR images.

\section{Inter-reader agreement by modality}

Inter-reader agreement of small opacity profusion treated dichotomously as positive (profusion $1 / 0$ or greater) versus negative $(0 / 0$ or $0 / 1)$ was similar overall for FSR $(\kappa 0.39,95 \%$ CI $0.28-0.49$ ) and CR ( $\kappa 0.42,95 \%$ CI 0.31-0.53) (table 3). Figure 2 presents 20 of the 28 reader-pair inter-reader kappa statistics (eight reader pairings had unstable kappa values due to high levels of agreement on 0/0 images). In general, reader agreement appeared similar between modalities (i.e. if a pair of readers had good agreement on FSR determinations they also tended to have good agreement on CR). For the 28 reader pairs, median kappa values for FSR (0.25) did not significantly differ from CR (0.30; Wilcoxon rank sum $\mathrm{p}=0.66)$, although readers tended to have slightly better agreement using CR. The same trend was observed for the mean kappa values with no statistical difference observed between mean FSR (0.31) and CR (0.36) kappa values $(\mathrm{p}=0.58)$.

\section{Small opacity shape/size designations between modalities}

The frequencies and percentages of the shape and size designations of small opacities are presented in figure 3 . The distribution of size classifications did not differ between FSR and CR. However, there was a significant difference between modality with respect to the classification of opacity shape.
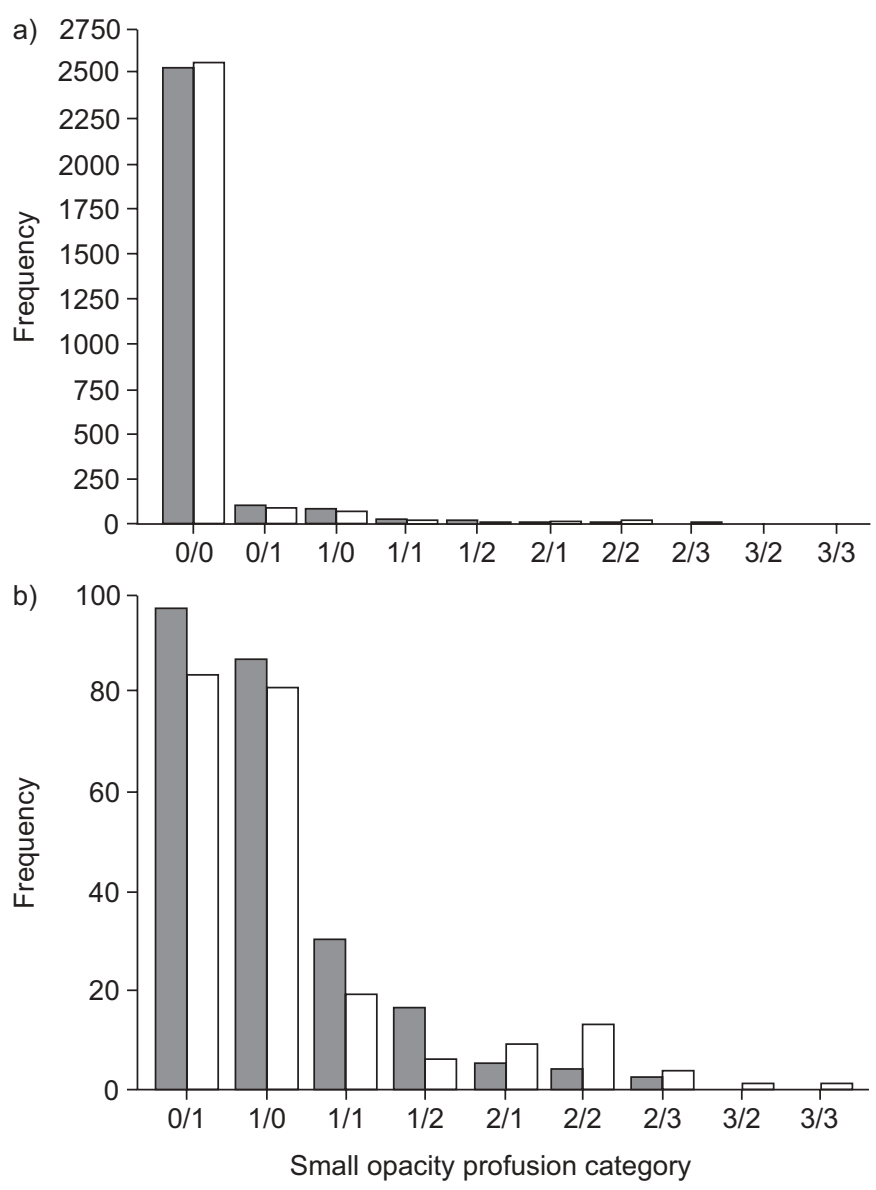

FIGURE 1. Profusion of small opacities for 1,388 radiographs, by imaging modality. Each image was classified by two B readers. $\square$ : traditional film-screen radiology; $\square$ : storage phosphor computed radiography.

More irregular opacities (compared to rounded) were classified using CR images compared to FSR (OR 1.8, 95\% CI 1.2-2.6; $\mathrm{p}=0.004)$. Inter-reader agreement was high with regards to characterisation of primary shape (irregular versus rounded) for both modalities, with $\kappa 0.91,95 \%$ CI $0.80-1.0$ for FSR and $\kappa 0.87,95 \%$ CI $0.72-1.0$ for CR.

\section{Pleural abnormalities}

Pleural abnormalities, including plaques, costophrenic angle obliteration, and diffuse pleural thickening were assessed. B readers classified 59 FSR images $(2.1 \%)$ as having a pleural abnormality consistent with pneumoconiosis, compared to 49 $(1.8 \%)$ CR images. Between-modality (within-reader) agreement was marginal ( $\kappa 0.38,95 \%$ CI $0.26-0.49)$ though better than between-reader agreement: between-reader agreement levels were low but similar for both FSR $(\kappa 0.23$, 95\% CI 0.08 $0.37)$ and CR ( $\kappa 0.18,95 \%$ CI $0.07-0.30)$.

\section{DISCUSSION}

Traditional film-screen chest radiographs have historically been invaluable in the investigation, assessment and monitoring of lung health. However, FSR is rapidly being replaced by various digital radiographic systems. Reading of hard copy films on view boxes has also been displaced by the interpretation of digital images displayed on computer monitors. The results of 
TABLE 2 International Labour Office small opacity profusion category by digital storage phosphor computed radiography (CR) and film-screen radiography (FSR) modalities

CR

Total

\begin{tabular}{|c|c|c|c|c|c|c|c|c|c|c|c|}
\hline & \multicolumn{10}{|c|}{ CR } & \multirow[t]{2}{*}{ Total } \\
\hline & $0 / 0$ & $0 / 1$ & $1 / 0$ & $1 / 1$ & $1 / 2$ & $2 / 1$ & $2 / 2$ & $2 / 3$ & $3 / 2$ & $3 / 3$ & \\
\hline \multicolumn{12}{|l|}{ FSR } \\
\hline $0 / 0$ & 2432 & 63 & 36 & 4 & & & & & & & 2535 \\
\hline $0 / 1$ & 69 & 7 & 19 & 2 & & & & & & & 97 \\
\hline $1 / 0$ & 50 & 10 & 19 & 3 & 2 & 1 & 2 & & & & 87 \\
\hline $1 / 1$ & 7 & 4 & 4 & 4 & 1 & 4 & 5 & 1 & & & 30 \\
\hline $1 / 2$ & & & 3 & 5 & 2 & 1 & 4 & 1 & & & 16 \\
\hline $2 / 1$ & & & & & & 2 & 1 & 1 & & 1 & 5 \\
\hline $2 / 2$ & & & & 1 & & & 1 & 1 & & 1 & 4 \\
\hline $2 / 3$ & & & & & 1 & 1 & & & & & 2 \\
\hline $3 / 2$ & & & & & & & & & & & 0 \\
\hline $3 / 3$ & & & & & & & & & & & 0 \\
\hline Total & 2558 & 84 & 81 & 19 & 6 & 9 & 13 & 4 & 0 & 2 & 2776 \\
\hline
\end{tabular}

this study indicate that, with appropriate attention to image acquisition and display, radiographs obtained using the widely available storage phosphor CR systems and displayed as soft copies can be equivalent to FSR with respect to image quality and the recognition of small parenchymal lung opacities.

We examined 1,388 working coal miners using a CR system in a mobile X-ray unit, and demonstrated satisfactory image quality and similarity of the two imaging modalities for the key outcome of prevalence of small interstitial lung opacities (ILO category $1 / 0$ or greater). In addition, imaging modality did not affect inter-reader agreement. However, the current study did demonstrate some differences between the CR and FSR images. The smallest opacities seen on CR soft copy images were more often identified by readers as irregular

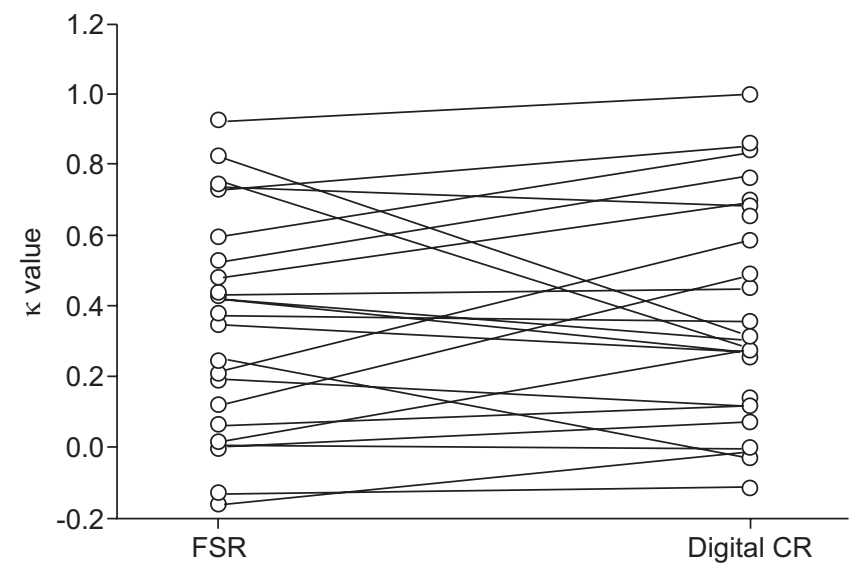

FIGURE 2. Inter-reader agreement in assessing the prevalence of pneumoconiosis (International Labour Office small opacity category $1 / 0$ or greater). Values are weighted kappas for 40-72 image pairs for 20 pairs of readers. Eight reader pairings not shown had unstable kappa values due to high levels of agreement on 0/0 images. Film-screen radiography (FSR) median (range) $\kappa 0.25(-0.17-0.92)$; digital storage phosphor computed radiography $(C R) \kappa 0.30(-0.01-1.0)$. (s-type), as compared to rounded (p-type) opacities recorded on FSR. The data also suggest that the readers identified a greater profusion of small opacities using CR images compared to FSR, but this difference was only seen in individuals with higher degrees of profusion (e.g. category $2 / 1$ or greater). Although these differences should not prevent adoption of $C R$ technology, additional research will be required to define the importance of these findings.

Two previous investigations have used human subjects in addressing the utility of digital images in the recognition of dust-related lung diseases $[3,10]$. In the study of TAKASHIMA et al. [10], readers evaluated laser-printed hard copies of digital images obtained using both CR and DR systems, as well as traditional FSR obtained within 6 months of the digitally acquired images

\begin{tabular}{|c|c|c|}
\hline \multirow[t]{3}{*}{ TABLE 3} & \multicolumn{2}{|c|}{$\begin{array}{l}\text { Inter-reader agreement within modality for the } \\
\text { presence of pneumoconiosis }\end{array}$} \\
\hline & & Second reader \\
\hline & CWP+ & CWP- \\
\hline \multicolumn{3}{|l|}{$\mathrm{FSR}^{\#}$} \\
\hline \multicolumn{3}{|l|}{ First reader } \\
\hline CWP+ & 30 & 56 \\
\hline CWP- & 28 & 1274 \\
\hline \multicolumn{3}{|l|}{ Digital CR } \\
\hline \multicolumn{3}{|l|}{ First reader } \\
\hline CWP+ & 30 & 38 \\
\hline CWP- & 36 & 1284 \\
\hline
\end{tabular}

Coal workers' pneumoconiosis (CWP) was defined as International Labour Office small opacity category $1 / 0$ or greater. FSR: film-screen radiography: CR: storage phosphor computed radiography. ${ }^{\#}$ : $\kappa 0.39$ (95\% Cl 0.28-0.49), agreement $0.94{ }^{\circ}:$ : 0.42 (95\% Cl 0.31-0.53), agreement 0.95 . 

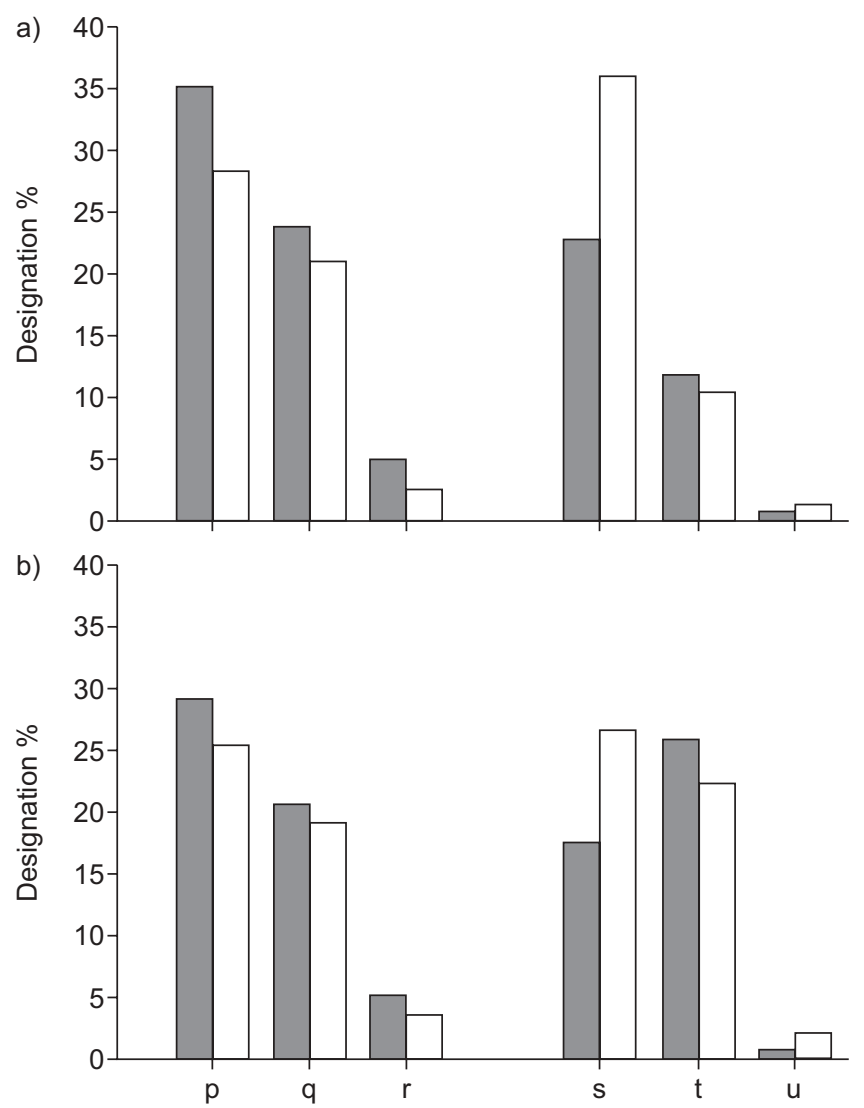

FIGURE 3. a) Primary and b) secondary small opacity shape and size designation by radiographic modality for films with International Labour Office small opacity category $1 / 0$ or greater: 1 : traditional film-screen radiology; $\square$ : storage phosphor computed radiography

among 20 silica-exposed workers and 10 healthy controls. Readers simultaneously viewed and compared the three hard copy images from each participant (FSR, CR, and DR) side-byside on view boxes. Some CR images were judged to show slightly lower profusion than the corresponding FSRs and this was also observed among DR images. The authors did suggest that the differences could be fixed with image modification.

FRANZBLAU et al. [3] compared ILO classifications by six B readers of traditional FSR and same-day DR images, displayed as soft copies from 107 subjects. Based upon their findings, these authors concluded that both FSR and DR can be recommended for the recognition and classification of dust-related parenchymal abnormalities. Images were rated without technical defects (ILO quality category 1 or 2) for $91 \%$ of soft copy DR images compared with $92 \%$ for FSR. These results, taken with ours, suggest that current digital systems are capable of providing soft copy images whose quality is judged at least equal to FSR.

DR systems have generally produced superior image quality compared to CR [2,11-15], although prior studies have often used simulated disease rather than actual human subjects in clinically relevant settings. Because CR systems are relatively inexpensive, more portable and versatile, they are presently in more common use than DR, but have not been extensively studied for interstitial lung disease. Several smaller patient studies have compared full-size hard copies or soft copy displays of CR images to FSR images, and recognition of small opacities appeared to be similar [13-15].

Taken together, the results of this study provide additional evidence that, with appropriate attention to image acquisition and soft copy display, the presence of small interstitial lung opacities on the chest radiographs of dust-exposed workers is equivalent, whether using contemporary approaches to digital chest imaging or traditional FSR. We also observed that intrareader agreement across imaging modalities was better than inter-reader agreement within modalities. This suggests that any lack of agreement in our study is due to differences in reading practices not related to the imaging modalities. Intrareader variation is also a criterion of interest and importance, but we were unable to assess it in the current study. Although the number of individuals in our study with higher degrees of profusion was small, readers appeared to identify a greater profusion of small opacities using CR images compared with FSR. Further studies among populations with a greater proportion of abnormal radiographs showing a high degree of profusion will be required to confirm this finding.

The study has a number of strengths. The results are based upon screening of a large number $(1,388)$ of dust-exposed workers, and there was no pre-selection of participants that could have affected the pre-test probability of abnormalities. Chest radiographs were collected in 2007, reflecting contemporary radiographic techniques. Each image was independently classified by two experienced NIOSH B readers, yielding 5,552 total readings. Standardised methodologies were used for acquisition and display of both the digital and traditional radiographs. The inter-reader agreement observed was similar to that in other studies [16-18].

There are also a number of study limitations. We were unable to draw firm conclusions regarding visualisation of large opacities between modalities because of the small number of large opacities observed. Pleural abnormalities were also sparse in our study, but a slight excess was noted for FSR over CR (2.1\% compared with $1.8 \%)$. The kappa values we report for pleural abnormalities between image modalities may be unstable, and these results alone are not adequate for the provision of guidance regarding the use of CR images in the recognition of pleural disease $[19,20]$. However, in agreement with our findings, FRANZBLAU et al. [3] also found more pleural disease with FSR compared with CR in a population with higher prevalence of pleural abnormality.

In general, as the prevalence of a condition under review approaches zero or $100 \%$, the kappa statistic may become unstable. For unweighted kappa values in the present study, we defined CWP as greater than or equal to the 1/0 ILO small profusion category. This yielded prevalence of $5.2 \%$ for FSR and $4.8 \%$ for CR. Because we chose a priori to include all images contributed by participants during the course of our routine surveillance, since we specifically wished to evaluate digital/film differences in a working monitoring programme, our prevalence was naturally constrained. Manipulation of the proportion of normal/abnormal images could lead to more stable kappa values, but the results would no longer be pertinent to a typical worker medical monitoring programme. 
An additional limitation of this and other studies that compare digital to traditional radiographs is the continuing evolution and diversity of imaging hardware and software. The images classified in this study did not rely on extensive image enhancement software; nevertheless, the findings are most relevant to images obtained and displayed using comparable methods, hardware and software. As digital imaging technologies continue to evolve, additional studies will be needed to define any impacts of these factors on the recognition and classification of dust-related abnormalities. Finally, the study objective was limited to assessing equivalence of imaging modalities. No independent indicator of disease was available, such as CT scan or lung biopsy, that could corroborate whether FSR or CR images more closely reflect the type and/or severity of lung or pleural pathology.

In conclusion, the current study, taken with the results of research undertaken to date, confirms that contemporary highquality digitally acquired chest images, displayed on calibrated medical-grade grayscale monitors, provide recognition of small interstitial opacities equal to those from FSR. Further studies are needed with respect to the recognition of large opacities and pleural abnormalities. Ultimately, the point may not be the equivalence of digital and FSR images, but whether digital technology can provide images that are superior for the identification and assessment of interstitial lung and pleural diseases.

\section{SUPPORT STATEMENT}

The findings and conclusions in this report are those of the authors and do not necessarily represent the views of the National Institute for Occupational Safety and Health (NIOSH). Mention of product names does not imply endorsement by NIOSH/Centers for Disease Control and Prevention.

\section{STATEMENT OF INTEREST}

None declared.

\section{ACKNOWLEDGEMENTS}

The authors wish to acknowledge the contribution of the National Institute for Occupational Safety and Health (Morgantown, WV, USA), the Coal Workers' Health Surveillance Program and the B readers to this study.

\section{REFERENCES}

1 International Labour Office. Guidelines for the Use of the ILO International Classification of Radiographs of Pneumoconioses. Geneva, International Labour Office, 2002.

2 Schaefer-Prokop C, Neitzel U, Venema HW, et al. Digital chest radiography: an update on modern technology, dose containment and control of image quality. Eur Radiol 2008; 18: 1818-1830.

3 Franzblau A, Kazerooni EA, Sen A, et al. Comparison of digital radiographs with film radiographs for the classification of pneumoconiosis. Acad Radiol 2009; 16: 669-677.
4 Centers for Disease Control and Prevention. Pneumoconiosis prevalence among working coal miners examined in federal chest radiograph surveillance programs - United States, 1996-2002. MMWR Morb Mortal Wkly Rep 2003; 52: 336-340.

5 Centers for Disease Control and Prevention. Pneumoconiosis in coal miners: NIOSH research and surveillance. MMWR Morb Mortal Wkly Rep Surveill Summ 1983; 32: 39SS-42SS.

6 Attfield MD, Castellan RM. Epidemiological data on US coal miners' pneumoconiosis, 1960 to 1988. Am J Public Health 1992; 82: 964-970.

7 Antao VC, Petsonk EL, Sokolow LZ, et al. Rapidly progressive coal workers' pneumoconiosis in the United States: geographic clustering and other factors. Occup Environ Med 2005; 62: 670-674.

8 American College of Radiology. Practice Guideline for Digital Radiography, Section IV. D. Display Capabilities (Res 42). Reston, American College of Radiology, 2007.

9 Cicchetti DV, Allison T. A new procedure for assessing reliability of scoring EEG sleep recordings. Am J EEG Technol 1971; 11: 101-109.

10 Takashima Y, Suganuma N, Sakurazawa H, et al. A flat-panel detector digital radiography and a storage phosphor computed radiography: screening for pneumoconioses. J Occup Health 2007; 49: 39-45.

11 Bacher K, Smeets P, Bonnarens K, et al. Dose reduction in patients undergoing chest imaging: digital amorphous silicon flat-panel detector radiography versus conventional film-screen radiography and phosphor-based computed radiography. AJR Am J Roentgenol 2003; 181: 923-929.

12 Biemans JM, Van Heesewijk JP, Van Der Graaf Y. Digital chest imaging: selenium radiography versus storage phosphor imaging. Comparison of visualization of specific anatomic regions of the chest. Invest Radiol 2002; 37: 47-51.

13 Fernandez JM, Ordiales JM, Guibelalde E, et al. Physical image quality comparison of four types of digital detector for chest radiology. Radiat Prot Dosimetry 2008; 129: 140-143.

14 Ganten M, Radeleff B, Kampschulte A, et al. Comparing image quality of flat-panel chest radiography with storage phosphor radiography and film-screen radiography. AJR Am J Roentgenol 2003; 181: 171-176.

15 Kroft LJ, Veldkamp WJ, Mertens BJ, et al. Comparison of eight different digital chest radiography systems: variation in detection of simulated chest disease. AJR Am J Roentgenol 2005; 185: 339-346.

16 Mannino DM, Kennedy RD, Hodous TK. Pneumoconiosis: comparison of digitized and conventional radiographs. Radiology 1993; 187: 791-796.

17 Naidoo RN, Robins TG, Solomon A, et al. Radiographic outcomes among South African coal miners. Int Arch Occup Environ Health 2004; 77: 471-481.

18 Welch LS, Hunting KL, Balmes J, et al. Variability in the classification of radiographs using the 1980 International Labor Organization Classification for Pneumoconioses. Chest 1998; 114: 1740-1748.

19 Thompson WD, Walter SD. Kappa and the concept of independent errors. J Clin Epidemiol 1988; 41: 969-970.

20 Thompson WD, Walter SD. A reappraisal of the kappa coefficient. J Clin Epidemiol 1988; 41: 949-958. 